\title{
Identification of genes involved in radioresistance of nasopharyngeal carcinoma by integrating gene ontology and protein-protein interaction networks
}

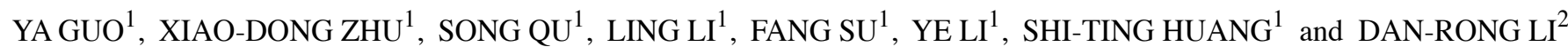 \\ ${ }^{1}$ Department of Radiation Oncology, Cancer Hospital of Guangxi Medical University, Cancer Institute of Guangxi \\ Zhuang Autonomous Region; ${ }^{2}$ Guang xi Medical Scientific Research Center, \\ Guangxi Medical University, Nanning 530021, P.R. China
}

Received June 15, 2011; Accepted July 29, 2011

DOI: $10.3892 /$ ijo.2011.1172

\begin{abstract}
Radioresistance remains one of the important factors in relapse and metastasis of nasopharyngeal carcinoma. Thus, it is imperative to identify genes involved in radioresistance and explore the underlying biological processes in the development of radioresistance. In this study, we used cDNA microarrays to select differential genes between radioresistant CNE-2R and parental CNE-2 cell lines. One hundred and eighty-three significantly differentially expressed genes $(\mathrm{p}<0.05)$ were identified, of which 138 genes were upregulated and 45 genes were downregulated in CNE-2R. We further employed publicly available bioinformatics related software, such as GOEAST and STRING to examine the relationship among differentially expressed genes. The results show that these genes were involved in type I interferon-mediated signaling pathway biological processes; the nodes tended to have high connectivity with the EGFR pathway, IFN-related pathways, NF- $\mathrm{B}$. The node STAT1 has high connectivity with other nodes in the protein-protein interaction (PPI) networks. Finally, the reliability of microarray data was validated for selected genes by semi-quantitative RT-PCR and Western blotting. The results were consistent with the microarray data. Our study suggests that microarrays combined with gene ontology and protein interaction networks have great value in the identification of genes of radioresistance in nasopharyngeal carcinoma; genes involved in several biological processes and protein interaction networks may be relevant to NPC radioresistance; in particular, the verified genes CCL5, STAT1- $\alpha$, STAT2 and GSTP1 may
\end{abstract}

Correspondence to: Dr Xiao-Dong Zhu, Department of Radiation Oncology, Cancer Hospital of Guangxi Medical University, Cancer Institute of Guangxi Zhuang Autonomous Region, 71 He Di Road, Nanning 530021, P.R. China

E-mail: zhuxdonggxmu@yahoo.cn

Key words: nasopharyngeal carcinoma, microarray, radioresistance, gene ontology, protein-protein interaction networks become potential biomarkers for predicting NPC response to radiotherapy.

\section{Introduction}

Nasopharyngeal carcinoma (NPC) is a major malignant tumor of the head and neck region and is endemic to Southeast Asia, especially Guangdong and Guangxi Provinces, and the Mediterranean basin $(1,3)$. Radiotherapy is the major treatment modality for nasopharyngeal carcinoma (NPC). However, in some cases, it is radioresistant. Although microarray methods have been used to assess genes involved in radioresistance in a number of cell types, including cervical, pancreatic, oral, lung, and esophageal cancers (4-8), but using cDNA microarray to identify a set of radioresistance-related genes from the cell lines with the same genetic background is limited. So we designed this study to investigate genes and the biology process that may be involved in this resistance.

Various high-throughput experiments are becoming more and more important in this post-genomic era. However, highthroughput also indicates highly-complex and noise-generating, how to systematically investigate these data and avoid those that might be selected by chance becomes a challenge $(9,10)$.

Gene ontology consists of three independent ontologies: biological processes (BP), cellular components (CC) and molecular functions (MF) (11). It has been widely used for elucidating the biological meaning of results in high-throughput experiments, such as with microarray $(12,13)$. Recently, accumulated knowledge on molecular interaction networks in human cells such as protein-protein, and protein-DNA interactions has been utilized to predict disease genes (14-16).

In this study, we first established a radioresistant cell line named CNE-2R. Second, we identified differential expression of genes using microarray. Third, we examined gene ontology terms of these genes and further explored how strongly these genes interact with each other in the whole proteininteraction networks. Finally, we verified partial differential gene in the mRNA and protein level using semi-quantitative RT-PCR and Western blotting. This biology-based investigation provides insight into the molecular mechanism of NPC radioresistance. 


\section{Materials and methods}

Cell lines and cell culture. CNE-2, a human NPC cell line, was purchased from cancer hospital of Shanghai Fudan University, and was grown in RPMI-1640 medium (Hyclone, USA) supplemented with $15 \%$ fetal calf serum (Gibco, USA), and maintained at $37^{\circ} \mathrm{C}$ in a humidified incubator with $5 \% \mathrm{CO}_{2}$.

Generation of radioresistant cell lines. CNE-2 cells were plated in $75 \mathrm{~cm}^{2}$ culture flask and irradiated with $400 \mathrm{cGy}{ }^{60} \mathrm{Co} \gamma$ radiation (THERATRONics780, Canada) when the cell grew to $~ 80 \%$ confluence. The culture medium was changed after irradiation and then the cells were again incubated. CNE-2 was treated with $400 \mathrm{cGy}$ again when it reached $\sim 80 \%$ confluence. These procedures were repeated 16 times to the total dose 64 Gy for 1 year.

Cloning formation assay for radiosensitivity. For cloning assay, cells were plated onto a 6-well plate and were irradiated with $\mathrm{X}$-rays at room temperature. The cells were exposed to doses of $0,0.5,1,2,4$ and $6 \mathrm{~Gy}$. After irradiation, the cells were cultured for 10 days in $5 \% \mathrm{CO}_{2}$ atmosphere at $37^{\circ} \mathrm{C}$. The colonies were fixed with carbinol (Kelong Chemical Reagent Factory, Cheng Du) and stained with $0.1 \%$ Giemsa (Solarbio, Pe King). Colonies containing $>50$ cells were scored as survivors. All experiments were performed three times. The survival fraction was calculated as the experimental group numbers of colonies divided by the numbers of cells seeded times plating efficiency. Plating efficiency was calculated as the control group numbers of colonies divided the numbers of cells seeded times $100 \%$. Cloning formation experiments were repeated three times. The dose-responses were analyzed using a linear-quadratic relationship model (LQ model): $\mathrm{SF}=\mathrm{e}^{\left({ }^{(} \mathrm{aD}+\beta \mathrm{DD} 2\right)}$. Graphpad Prism 4 software was used to create fit cure, where $\mathrm{D}$ is the single radiation dose and $\mathrm{SF}$ is the surviving fraction at dose $\mathrm{D}, \alpha$ and $\beta$ are radiobiological parameters.

Whole human genome microarray and data analysis. All experiments were performed by Illumina Human-6v3.0 microarray from Shanghai Biochip Co.,Ltd. Each microarray which can detect six samples at the same time, consists of 48000 transcripts. The whole gene chip experiments were repeated. Total RNA was, respectively, extracted from the CNE-2R and CNE-2 with TRIzol reagent (Invitrogen) following the manufacturer's instructions. The concentration of RNA was validated with a UV spectrophotometer at $260 / 280 \mathrm{~nm}$. The integrity of RNA was measured by agarose gel electrophoresis. RNA was synthesized by reverse transcription (RT) and purified by Illumina Total Prep RNA Amplification Kit (Cat1791). The chip was scanned with Illumina Bead Chip Reader. Spot and background intensities were acquired with Bead Station software. The data were analyzed by Bead Studio software. Differential gene screening criteria: the experimental group or control group contain effective genes $(\mathrm{p}<0.01)$ and single sample differscore value in the experimental group was $<-20$ or $>+20$. Significant difference of genes were analyzed by GEPAS (17-19). A t-test was used for the difference in mean expression between the two groups of arrays. t-statistics and p-values are reported. Genes are ranked according to the t-statistics. Genes at the top of the results list are those more expressed in the first class. Genes at the bottom part of the list are those more expressed in the second class.

Gene ontology enrichment analysis and visualization. We applied Gene Ontology Enrichment Analysis Software Toolkit (GOEAST) (11) to explore the functional characteristics of the radioresistance-related genes in NPC. During the data process, 138 increased genes in CNE-2R were submitted to the GOEAST as the test genes and the control genes as the reference genes. For each GO term, Fisher's exact test was performed to detect whether the GO term is significantly overrepresented among the genes in a gene list by comparing to the control genes. P-value from Fisher's exact test was $<1.0 \times 10^{-3}$. The smaller the p-value is, the more significant the GO term is enriched in the dataset.

Protein networks encoded by the significant genes. Proteinprotein interaction networks were done by STRING online software (http://string.embl.de) through inputting ID number $(20,21)$. The following two criteria were applied to detect important nodes: i) required confidence: high confidence (score: 0.07), ii) interactors: no more than 5 .

RNA extraction and RT-PCR analysis. Total RNA was extracted from cell lines with the TRIzol reagent (Invitrogen, USA). Reverse-transcribed with MLV reverse transcriptase (Promega, USA) and random primers (Takara). PCR was performed in $25 \mu 1$ reaction volume. Three randomly selected genes (STAT1, CCL5 and GSTP1) differentially expressed in CNE-2R compared with CNE-2 according to our microarray data and an invariant housekeeping gene control GAPDH, were amplified according to the designed primers (Shanghai Sangon Biotechcol., Ltd.). Primers sequences are presented in Table I. PCR products were analyzed by electrophoresis on $1.5 \%$ agarose gel containing ethidium bromide. All experiments were performed at least three times. The intensity of each band was quantitated by the Quantity one software. Semi-quantitative RT-PCR results are expressed as the mean $\pm \mathrm{SD}$ value. $\mathrm{P}<0.05$ was considered statistical significance.

Western blot analysis for STAT1 and STAT2. Total cellular protein was, respectively, extracted from CNE-2R and CNE-2. The protein concentration of each sample was determined by Bradford assay (Beyotime, China). Proteins were separated on 10\% SDS-PAGE and transferred to PVDF membranes (Solarbio, PeKing). STAT $1-\alpha / \beta$ and STAT2 were detected with mouse anti-STAT1 P84/P91 protein antibody and rabbit anti-STAT2 protein antibody (Cell Signaling Technology), respectively. Then the blot was incubated with a secondary antibody, IRDye ${ }^{\text {TM }} 700 \mathrm{DX}$ conjugated affinity purified (red fluoresence) anti-mouse or IRDye ${ }^{\mathrm{TM}} 700 \mathrm{DX}$ conjugated affinity purified (green fluoresence) anti-rabbit fluoresence recommended by manufactures (KPL Co., USA). Images of STAT1 and STAT2 were obtained using the infrared fluorescence imaging system (Odyssey, Licor Co., USA). GAPDH was used as a loading control. All experiments were performed at least three times. The densities of protein bands were calculated using Quantity one software, and the experiments repeated three times. Significant difference was estimated by Student's twotailed t-test with cut-off $\mathrm{p}<0.05$. 
Table I. RT-PCR primers.

\begin{tabular}{|c|c|c|}
\hline Gene & Primer sequences & $\begin{array}{l}\text { Length } \\
\text { (bp) }\end{array}$ \\
\hline CCL5 & $\begin{array}{l}\text { СCTCATTGCTACTGCCCTCTGCG } \\
\text { AАСТССТGACСТCAAGTGATCCACC }\end{array}$ & 477 \\
\hline STAT1 & $\begin{array}{l}\text { TACTTTCCCTGACATCATTCG } \\
\text { ATTCTACAGAGCCCACTATCC }\end{array}$ & 302 \\
\hline GSTP1 & $\begin{array}{l}\text { CCGTGGTCTATTTCCCAGTTCG } \\
\text { GTGCCTTCACATAGTCATCCTTGC }\end{array}$ & 354 \\
\hline GAPDH & $\begin{array}{l}\text { GAAGGTGAAGGTCGGAGT } \\
\text { GAAGATGGTGATGGGATTTC }\end{array}$ & 225 \\
\hline
\end{tabular}

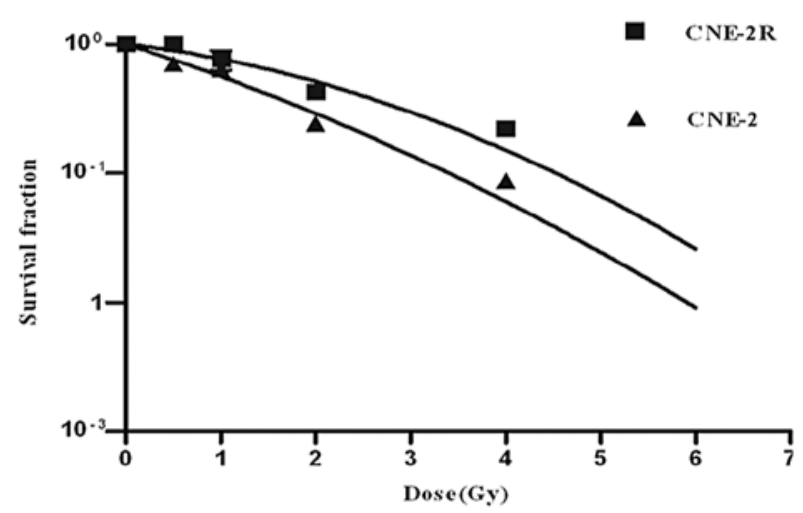

Figure 1. Survival curves of parental CNE-2 cell lines and radioresistant CNE-2R cell lines. CNE-2 or CNE-2R cells were seeded onto 6-well plates and further irradiated with various doses (0-6 Gy). The cells were cultured for 10 days to allow colony formation. Because cells at $6 \mathrm{~Gy}$ were polluted, we could not see related information at $6 \mathrm{~Gy}$ in (A) and (B).

\section{Results}

Radiation sensitivity of different radioresistant cell lines. We investigated the survival curves of parental CNE-2 cell lines and the radioresistant $\mathrm{CNE}-2 \mathrm{R}$ cell line by colony formation assay. The radiation dose-response of different cells are shown in Fig. 1, indicating that the CNE-2R survival curves are significantly higher than $\mathrm{CNE}-2$. The average X-ray survival curve parameters are given in Table II, $\alpha$ and $\beta$ parameters of the linear-quadratic relationship model were observed. CNE-2R and CNE-2 had different SF2, respectively, 0.499 and 0.284. Thus, SF2 in the CNE-2R is greater than in CNE-2. CNE-2R and CNE- $2 \alpha, \beta$ values are: 0.2769 and $0.03454,0.594$ and 0.03623 . $\alpha / \beta$ values are: 8.016 and $16.395 ; \alpha / \beta$ measure cell repair capacity. The high ratio indicates that the repair capacity was weak. Therefore, we can infer that $\mathrm{CNE}-2 \mathrm{R}$ repair capability is stronger than $\mathrm{CNE}-2$ repair capacity. All the above suggest that $\mathrm{CNE}-2 \mathrm{R}$ is radio-resistant.

Differentially expressed genes in CNE-2R. Both parental CNE-2 cell line and radioresistant $\mathrm{CNE}-2 \mathrm{R}$ cell line were subjected to cDNA microarray analysis. Comparing these two cell lines,
Table II. Correlation parameters in the linear-quadratic relationship model.

\begin{tabular}{lcccr}
\hline Cell lines & SF2 & $\alpha$ & $\beta$ & $\alpha / \beta$ \\
\hline CNE-2R & 0.499 & 0.2769 & 0.03454 & 8.016 \\
CNE-2 & 0.284 & 0.594 & 0.03623 & 16.395 \\
\hline
\end{tabular}

there were 596 different genes found in the first experiment, 870 genes were detected in the second experiment. Three hundred and forty-seven genes were differentially expressed in both experiments. We further used GEPAS to find the significant differences. One hundred and thirty-three genes had significant difference $(\mathrm{p}<0.05)$. There were 138 up-regulated and 45 downregulated genes in CNE-2R-radioresistant cell line (Fig. 2). The 183 significant genes might correlate with radioresistance, therefore we investigated their features in gene function and protein interaction networks.

Gene ontology results by GOEAST. The GO-term enrichment test is a useful approach in the functional analysis of a set of genes. GO represents the biological knowledge as a tree (more precisely as a directed acyclic graph, DAG, in which a node can have more that one parent). We submitted 138 up-regulated genes to GOEAST, 126 genes related with significant GO terms and 23 significant biological process were found that might be involved in radioresistance of NPC. The details of the biological process are shown in Fig. 3. Most of the significant biological processes in ontology terms was type I interferon-mediated signaling pathway $(p=2.54 \mathrm{E}-14)$. Seventeen genes had annotations of GO terms type I interferon-mediated signaling pathway, including IRF1, IFITM1, STAT1, XAF1, IFI27, IFITM3, IFIT2, IFIT1, SP100, STAT2, ISG15, OASL, IFI35, OAS2, IFIT3, IRF9, MX2. Most of these genes were over-expressed in CNE-2R except XAF1. Other enriched GO terms were related to cellular response to cytokine stimulus $(\mathrm{p}=5.65 \mathrm{E}-14)$, response to other organism $(\mathrm{p}=8.42 \mathrm{E}-14)$, response to virus $(\mathrm{p}=3.58 \mathrm{E}-13)$, immune system process $(\mathrm{p}=1.05 \mathrm{E}-13)$, response to stress $(\mathrm{p}=5.58 \mathrm{E}-11)$.

Protein-protein interaction analysis. To explore the protein interaction networks encoded by all significant genes, we extracted networks using the STRING.As shown in Fig. 4, 170 seed proteins were involved in protein-protein interactions. Of which 141 were up-regulated proteins, 39 were down-regulated proteins. In the network, up-regulated proteins usually interacter with down-regulated to consistute a big network, such as, STAT1FOS-MAPK4-GSTP1. These seed proteins have important functions in signal transduction, response to stimulation, cell proliferation, and anti-apoptosis, for example, FOS, HEY2, RHOQ, CDKN1A, IL-1 $\alpha$, JUN, CCL5, STAT1, and STAT2 are involved in signal transduction. KRT6A, CDKN1A, JUN, CCL5, STAT1, ZP4, IL-1 $\beta$, TNFAIP3 can positively regulate cell proliferation. CDKN1A, IL-1 $\alpha$, IL-1 $\beta$, CCL5, TNFAIP3, SOD2, THBS1 are involved in anti-apoptosis. Many proteins are closely related with STAT1, among them 6 nodes STAT2, RNF31, XAF1, IRF1, JUN, FOS highly relevant with STAT1 (confidence $>0.09$ ). Based on the above 


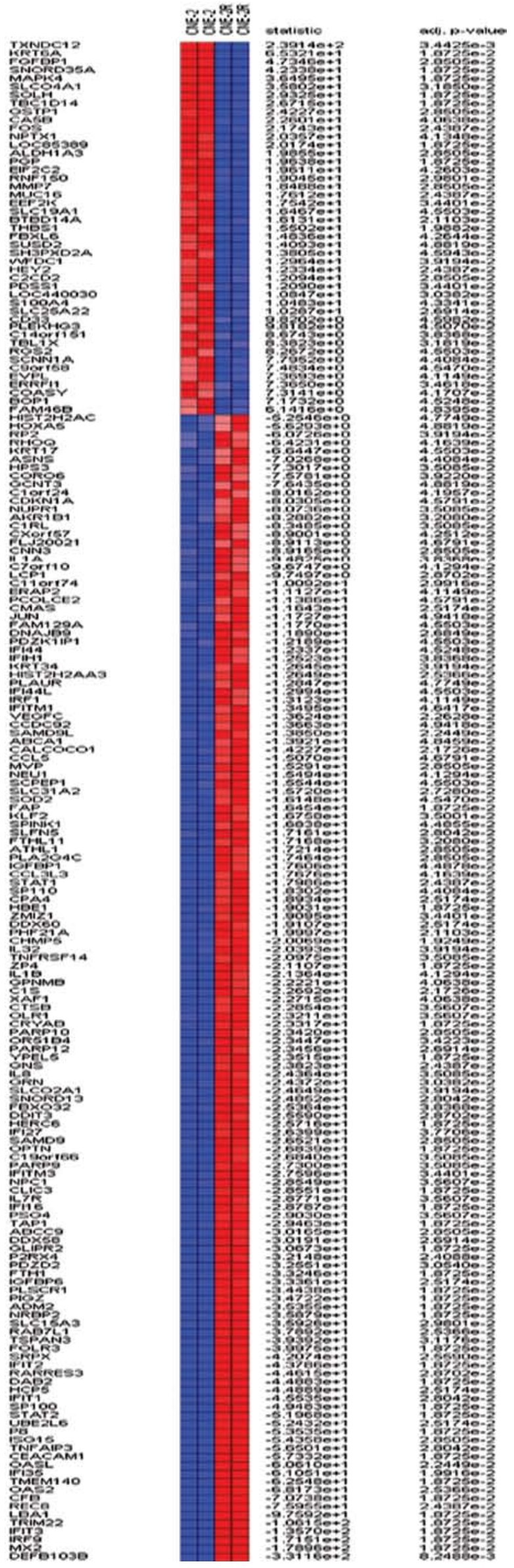

Figure 2. Different expression genes between CNE-2R and CNE-2. The left grid represents gene names; the right grid respectively estimated statistics for the difference in intensities and the adjusted p-values attached to each estimate of the statistics; on the top are the names of the arrays and their classes: CNE-2 and CNE-2R. Red squares represent genes having high expression. Blue squares represent lower expressions. Genes on the top of the results list are those more expressed in CNE-2. Genes on the bottomt of the list are those more expressed in CNE-2R. 


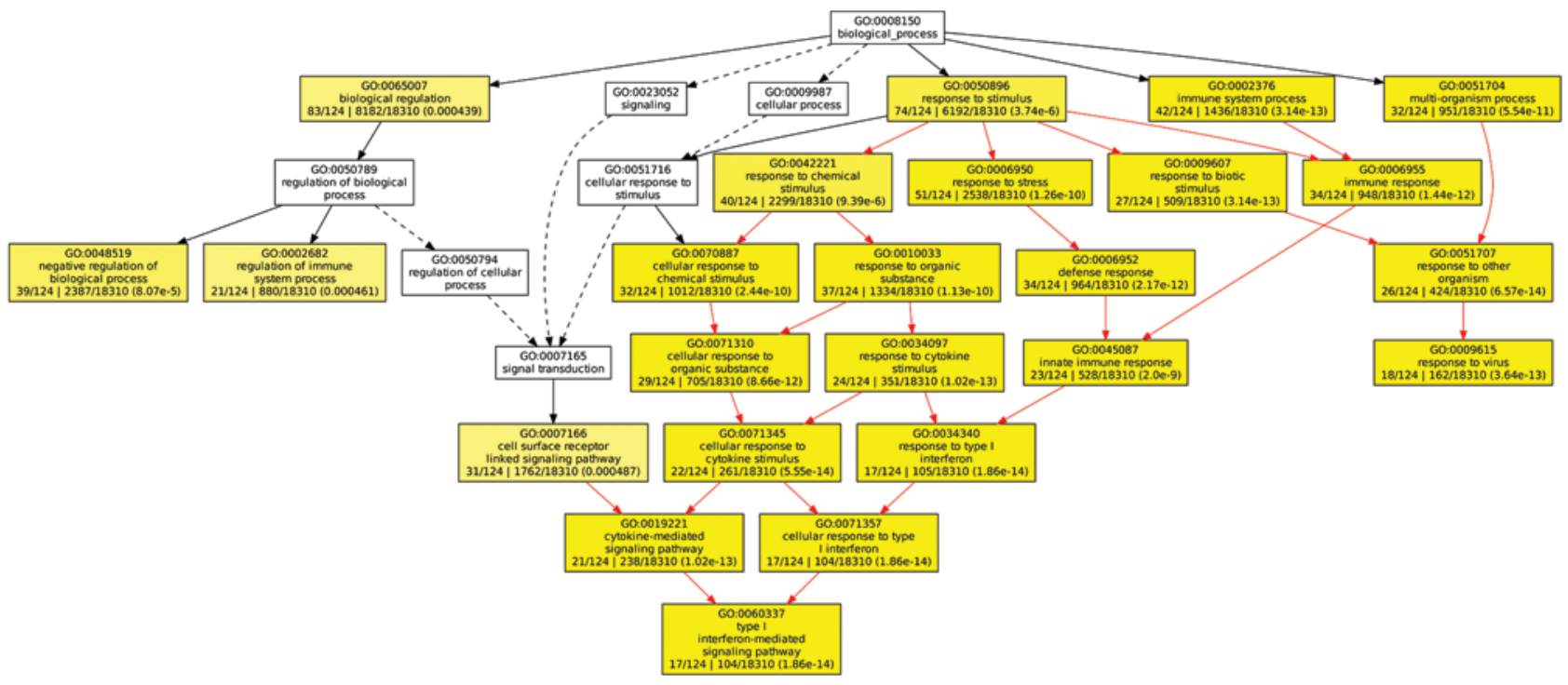

Figure 3. Significantly enriched GO terms in the biological process among 138 up-regulated genes. Boxes represent GO terms, labeled by its GOID, term definition, detailed information, organized as q/m/t/k (p-value). Significantly enriched GO terms are marked yellow. The degree of color saturation of each node is positively correlated with the significance of enrichment of the corresponding GO term. Non-significant GO terms within the hierarchical tree are shown as white boxes. Edges stand for connections between different GO terms. Red edges stand for relationship between two enriched GO terms, black solid edges stand for relationship between enriched and unenriched terms, black dashed edges stand for relationship between two unenriched GO terms.

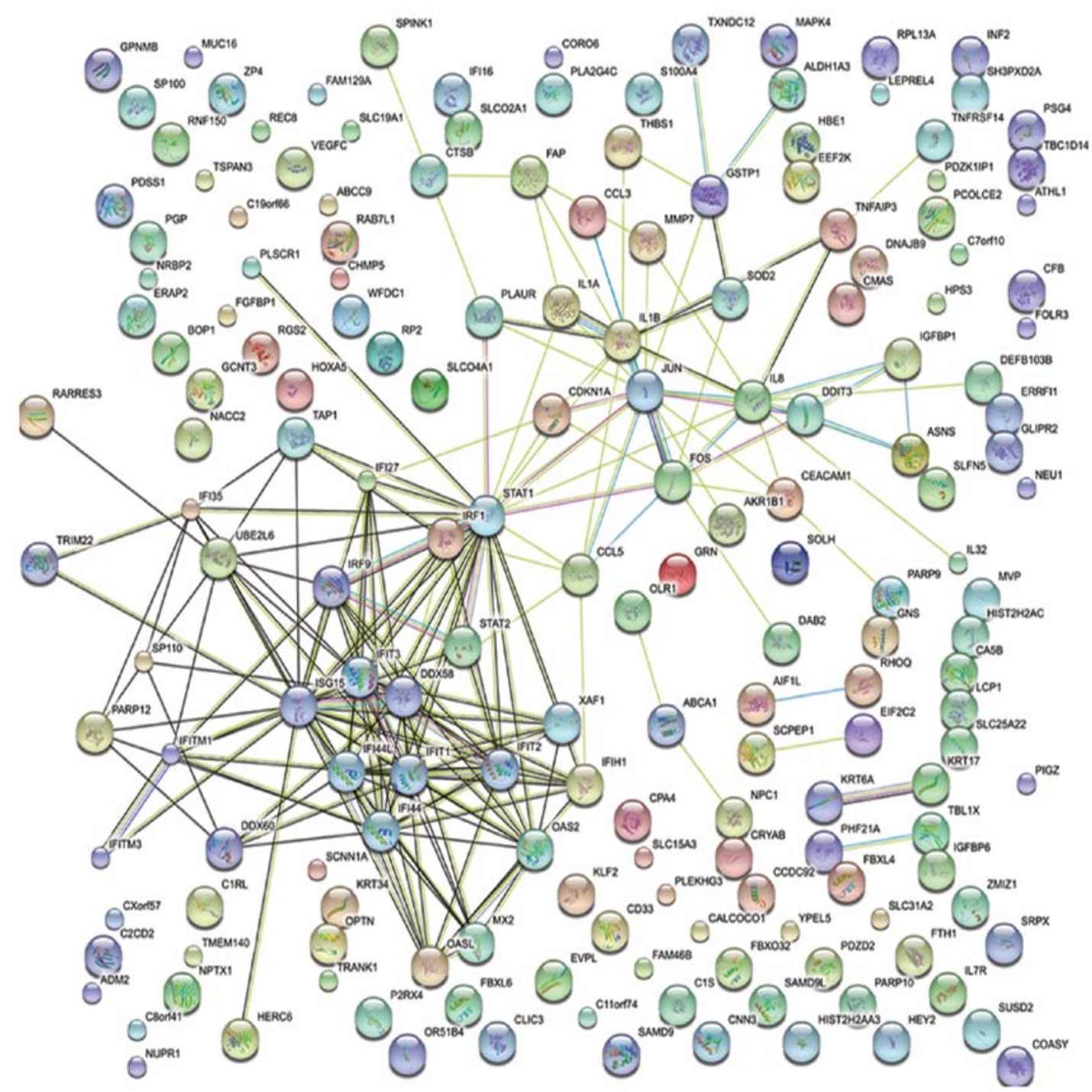

Figure 4. Protein-protein interaction networks analyzed by String software. Network display, nodes are colored (if they are directly linked to the input). 

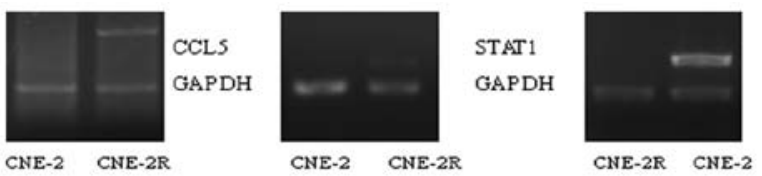

GSTP1 GAPDH

Figure 5. Verification of gene expressions by semi-quantitative RT-PCR. Up-regulated gene CCL5, STAT1 and down-regulated gene GSTP1 in the radioresistant $\mathrm{CNE}-2 \mathrm{R}$ cell line was detected by semi-quantitative RT-PCR. The internal standard (GAPDH) was amplified with each gene.

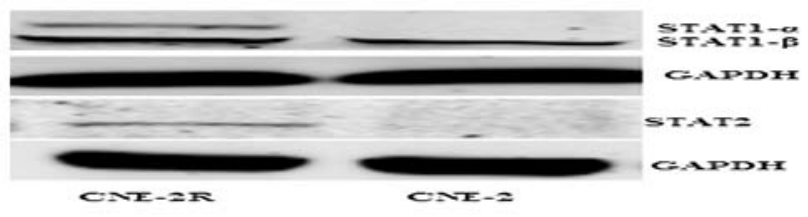

Figure 6. Western blot analysis for STAT1 and STAT2.

analysis, we used Western blotting to verify variation of STAT1 and STAT2 in protein level.

Validation of differential regulated genes by RT-PCR and Western blot analysis. We investigated two up-regulated genes CCL5, STAT1 and the down-regulated gene GSTP1 in radioresistant CNE-2R cell lines using RT-PCR to confirm the results of microarray screening. As shown in Fig. 5, CCL5 and STAT1 was only expressed in the CNE-2R cell line. In the CNE-2 cells the expression of CCL5 and STAT1 equal to zero $(\mathrm{p}<0.05)$. The expression of GSTP1 was much higher in CNE-2 than in CNE-2R cells. It was not expressed in CNE-2R cells $(\mathrm{p}<0.05)$. There was good agreement with our cDNA results in mRNA level $(\mathrm{p}=0.00000918,0.0017353$ and 0.000254 for CCL5, STAT1 and GSTP1, respectively).

To further evaluate the reliability of the microarray data, two genes, selected as representatives were analyzed by Western blotting. According to our array analysis, STAT1 and STAT2 were up-regulated in CNE-2R. Western blotting showed that the expression of STAT1- $\alpha$ in radioresistant CNE-2R cell line was greater than in the parental CNE-2 cell line, while the expression of STAT1- $\beta$ in these two cell lines showed no difference. The expression of STAT2 was obviously increased in CNE-2R, supporting the reliability of array data (Fig. 6).

\section{Discussion}

Radioresistance remains a major problem in the treatment of NPC (22-24). The molecular mechanisms are still unclear, and till now, there are no effective biomarkers for predicting NPC radiosensitivity. Traditional method to investigate radioresistantrelated genes may not be completely accurate and may be contradictory $(9,10)$.

In this study, we first constructed the radio-resistant cell line CNE-2R through giving CNE-2 cell line fractionated radiation in vitro; second, we used cDNA microarray to select the different genes between radioresistant CNE-2R and parental CNE-2 cell line. Third, we further employed GOEAST and STRING to examine the relationship among all differentially expressed genes instead of focusing on a handful of arbitrarily chosen 'candidate' genes. To confirm the changes in mRNA and protein level, we performed semi-quantitative RT-PCR and Western blotting. This biology-based investigation is helpful for finding biomarkers and elucidating the molecular mechanism of NPC radioresistance.

Using DNA microarray techniques it has been shown that STAT1 was consistently up-regulated after radiation by daily multi-fractions in breast cancer, prostate cancer and glioma, STAT1 over-expression has also been demonstrated in the radioresistance of head-and-neck cancer cell lines, leukemia, and multiple myeloma $(25,26)$; high levels of constitutively active STAT1 have also been reported to be indispensable to support cell survival in several different types of cancer. STAT1 and STAT2 belong to IFN-inducible pathways, STAT1 is the most highly expressed gene in the IFN-signaling pathway and it has been reported that exposure to IFN enhances cell survival after radiation $(27,28)$. Therefore, we inferred that STAT1, STAT2 might improve cell survival by activating IFN-inducible pathway. STAT1 is important for transducing pro-apoptotic signals due to transcriptional activation of genes or through a non-trans-criptional mechanism by inhibiting the anti-apoptotic protein NF-кB (29). STAT1 has been demonstrated to modulate the checkpoint cell cycle responses after DNA damage, STAT1-deficient cells show defects in both intraS-phase and G2/M checkpoints. These effects are associated with reduced functional activity of the ATM-Chk2 pathway in cells lacking STAT1 (30). The vast majority of cancer cells will arrest in different phases of the cell cycle after irradiation. This is thought to be the important self-protective mechanism to ensure the cells gain enough time to repair DNA damage before replication or cell mitosis (24). Our data showed that the expression of STAT1 and STAT2 are higher in CNE-2R than in CNE-2; in the GO category 'molecular function', STAT1 is involved in many significant biological processes, and has great value $(\mathrm{p}<0.001)$. In protein-protein interaction analysis, STAT1 correlated with 17 nodes, including tumor-associated genes (JUN, FOS), cell cycle related genes (CDKN1A) and negative regulation cell cycles genes (DDIT3, CDKN1A, IL-8); Western blot results indicate STAT1- $\alpha$ is high in the radioresistant CNE-2R cell line. Therefore, we speculated that highly expressed STAT1 and STAT2 might, through supporting cell survival, make CNE-2R radioresistant. Thus, STAT1 and STAT2 might become molecular targets for improving radiosensitivity of NPC.

Most existing radiation-resistance mechanisms indicate that radioresistance is associated with an up-regulation of DNA repair and stress response genes, down-regulation of cell cycle and apoptosis genes, immune response to organize genes (31). CDKN1A functions as a regulator of cell cycle progression at G1. The expression of this gene is tightly controlled by the tumor suppressor protein $\mathrm{p} 53$, through which this protein mediates the p53-dependent cell cycle G1 phase arrest in response to a variety of stress stimuli. This protein can interact with proliferating cell nuclear antigen (PCNA), a DNA polymerase accessory factor, and plays a regulatory role in S phase DNA replication and DNA damage repair. It is known that $\mathrm{S}$ phase cells are radioresistant. Thus, CDKN1A might be involved in radioresistance through adjusting cell cycle and DNA damage repair. Our GOEAST data clearly showed that all significant genes involved in 23 biological processes, including type I 
interferon-mediated signaling pathway, cellular response to cytokine stimulus, response to other organism, response to virus, immune system process, and response to stress. This result coincided with previous report. We searched 170 nodes from protein interaction nodes using STRING, these nodes are closely related (Fig. 4). ABCA1, CCL5, CDKN1A, DAB2, DDIT3, DDX58, IFI44L, IFIH1, IFIT1, IFIT2, IFIT1, IL-1 $\alpha$, IL- $\beta$, IL-8, ISG15, JUN, OASL, STAT1, STAT2, THBS1 both were in the GOEAST and STRING results. Integrating Microarray with gene ontology and protein interaction networks, showed that KRT6A, CDKN1A, JUN, CCL5, STAT1, ZP4, IL-1 $\beta$, TNFAIP3 can positively regulate cell proliferation, and up-regulated expression genes CDKN1A, IL-1 $\alpha$, IL-1 $\beta$, CCL5, TNFAIP3, SOD2, THBS1 are involved in anti-apoptosis. DDIT3, CDKN1A, IL-8 correlated with negative regulation of the cell cycle, and expression of these above-mentioned genes were increased in CNE-2R, this analysis further confirmed the view that radioresistance is associated with DNA damage repair, apoptosis, cell cycle checkpoints, and immune response.

IRF1, IFITM1, STAT1, XAF1, IFI27, IFITM3, IFIT2, IFIT1, SP100, STAT2, ISG15, OASL, IFI35, OAS2, IFIT3, IRF9, MX2 belong to type I interferon-mediated signaling pathway. Study has suggested that lack of interferon- $\beta$ induced radiosensitization in four out of five human glioblastoma cell lines, IFN enhances cell survival after radiation. Up-regulation of components of the IFN-related signaling pathway plays a role in tumor radioresistance $(27,28)$. Therefore, we can infer that up-regulated genes IFI44L, IFIH1, IFIT1, IFIT2, IFIT1, STAT1, STAT2, ISG15, OASL might relate with tumor radioresistance by promoting tumor cell survival. The IFN pathway might play a role in radioresistance of NPC. DDX58, IFIH1 can activate $\mathrm{NF}-\kappa \mathrm{B}$. The elevated basal NF- $\kappa \mathrm{B}$ activity in certain cancers has been linked with tumor resistance to chemotherapy and radiation (32). DDX58, IFIH1 might contribute to radioresistance by active NF- $\kappa$ B. STAT1-JUN-FOS belongs to EGF signaling pathway. The epidermal growth factor receptor-signaling pathway has been associated with tumor radioresistance and STAT1 is induced by radiation in association with epidermal growth factor receptor. EGFR has long been considered to play an important role in radiation response of epithelial, overexpression of EGFR in tumors correlating with poor response to radiotherapy and increasing level of EGFR correlating with radioresistance in head and neck cancer cell lines. Using antiEGFR monoclonal antibodies can improve radiosensitivity $(33,34)$. JUN is a proto-oncogene, expression of c-Jun promotes cellular survival by negatively regulating the expression of the tumor-suppressor PTEN (35). c-Jun is a major constituent of AP-1 transcription factor that transduces multiple mitogen growth signals (36). Expression of AP-1 proteins has been associated with a more aggressive clinical outcome in prostate cancer. Together, the findings show that AP-1 activity in prostate cancer cells mediates EGF-R and PI3K signalling, is essential for their proliferation, and confers protection against radiationinduced cell death. Thus, transcription factor AP-1 promotes growth and radioresistance in prostate cancer cells (37). STAT1JUN-FOS JUN might play an essential role in radioresistance through EGFR pathway or AP-1. By using STRING to elucidate potential molecular interactions and networks, we found that most genes differentially expressed in CNE-2R could be coupled to interferon $\alpha, \beta$, EGFR pathway, or indirectly active
$\mathrm{NF}-\kappa \mathrm{B}$. we further verified that radioresistance might correlate with active interferon $\alpha, \beta$, EGFR pathway.

In conclusion, we used microarray to identify 183 differential genes in the radioresistant NPC cell line CNE-2R. We further used gene ontology and protein interaction networks to analyze the 183 differential genes, showing that 23 biological processes might be relevant to NPC radioresistance; the verified genes CCL5, STAT1- $\alpha$, STAT2 and GSTP1 might become potential biomarkers for predicting NPC response to radiotherapy. These results could have clinical value in distinguishing radiosensitive from radioresistant NPC and could be helpful in personalized therapeutic strategies.

\section{Acknowledgements}

This study was supported by the Guangxi Natural Science Foundation (grant no. 0832229) and the National Natural Science Foundation of China Grant 30860329.

\section{References}

1. Jia WH, Huang QH, Liao J, Ye W, Shugart YY, Liu Q, Chen LZ, Li YH, Lin X, Wen FL, et al: Trends in incidence and mortality of nasopharyngeal carcinoma over a 20-25 year period (1978/19832002) in Sihui and Cangwu counties in southern China. BMC Cancer 6: 178-185, 2006.

2. Sun Y, Yi H, Yang Y, Yu Y, Ouyang Y, Yang F, Xiao Z and Chen Z: Functional characterization of p53 in nasopharyngeal carcinoma by stable shRNA expression. Int J Oncol 34: 1017-1027, 2009.

3. Fang WY, Li X, JIANG QP, Liu Z, Yang HL, et al: Transcriptional patterns, biomarkers and pathways characterizing nasopharyngeal carcinoma of Southern China. J Translat Med 6: 32-44, 2008.

4. Fukuda K, Sakakura C, Miyagawa K, Kuriu Y, Kin S, Nakase Y, Hagiwara A, Mitsufuji S, Okazaki Y, Hayashizaki Y, et al: Differential gene expression profiles of radioresistant oesophageal cancer cell lines established by continuous fractionated irradiation. Br J Cancer 91: 1543-1550, 2004.

5. Guo WF, Lin RX, Huang J,Zhou Z, Yang J, Guo GZ and Wang SQ: Identification of differentially expressed genes contributing to radioresistance in lung cancer cells using microarray analysis. Radiat Res 164: 27-35, 2005.

6. Wong YF, Sahota DS, Cheung TH, Lo KW, Yim SF, Chung TK, Chang AM and Smith DI: Gene expression pattern associated with radiotherapy sensitivity in cervical cancer. Cancer $\mathbf{J} 12$ : 189-193, 2006.

7. Ogawa K, Utsunomiya T, Mimori K, Tanaka F, Haraguchi N, Inoue $\mathrm{H}$, Murayama $\mathrm{S}$ and Mori M: Differential gene expression profiles of radioresistant pancreatic cancer cell lines established by fractionated irradiation. Int J Oncol 28: 705-713, 2006.

8. Ishigami T, Uzawa K, Higo M, Nomura H, Saito K, Kato Y, Nakashima D, Shiiba M, Bukawa H, Yokoe H, et al: Genes and molecular pathways related to radioresistance of oral squamous cell carcinoma cells. Int J Cancer 120: 2262-2270, 2007.

9. Beyer A, Bandyopadhyay S and Ideker T: Integrating physical and genetic maps: from genomes to interaction networks. Nat Rev Genet 8: 699-710, 2007.

10. Stumpf MP, Thorne T, De Silva E, Stewart R, An HJ, Lappe M and Wiuf C: Estimating the size of the human interactome. Proc Natl Acad Sci USA 105: 6959-6964, 2008.

11. Harris MA, Clark J, Ireland A, Lomax J, Ashburner M, Foulger R, Eilbeck K, Lewis S, Marshall B, Mungall C, et al: The Gene Ontology (GO) database and informatics resource. Nucleic Acids Res 32: D258-D261, 2004.

12. Mosquera JL and Sanchez-Pla A: SerbGO: searching for the best GO tool. Nucleic Acids Res 36: W368-W371, 2008.

13. Zheng Q and Wang XJ: GOEAST: a web-based software toolkit for Gene Ontology enrichment analysis. Nucleic Acids Res 36: W358-W363, 2008.

14. Nakou M, Bertsias G, Stagakis I, Centola M, Tassiulas I, Hatziapostolou M, Kritikos I, Goulielmos G, Boumpas DT and Iliopoulos D: Gene network analysis of bone marrow mononuclear cells reveals activation of multiple kinase pathways in human systemic lupus erythematosus. PLoS One 5: e13351-e13360, 2010. 
15. Oldham MC, Horvath S and Geschwind DH: Conservation and evolution of gene coexpression networks in human and chimpanzee brains. Proc Natl Acad Sci USA 103: 17973-17978, 2006.

16. Ortutay $\mathrm{C}$ and Vihinen $\mathrm{M}$ : Identification of candidate disease genes by integrating Gene Ontologies and protein-interaction networks: case study of primary immunodeficiencies. Nucleic Acids Res 37: 622-628, 2009.

17. Montaner D, Tarraga J,Huerta-Cepas J, Burguet J, Vaquerizas JM, Conde L, Minguez P, Vera J, Mukherjee S, Valls J, et al: Next station in microarray data analysis: GEPAS. Nucleic Acids Res 34: W486-W491, 2006.

18. Al-Shahrour F, Minguez P, Tarraga J, Montaner D, Alloza E, Vaquerizas JM, Conde L, Blaschke C, Vera J and Dopazo J: BABELOMICS: a systems biology perspective in the functional annotation of genome-scale experiments. Nucleic Acids Res 34: W472-W476, 2006.

19. Keijser BJ, Ter Beek A, Rauwerda H, Schuren F, Montijn R, van der Spek H and Brul S: Analysis of temporal gene expression during Bacillus subtilis spore germination and outgrowth. J Bacteriol 189: 3624-3634, 2007.

20. Szklarczyk D, Franceschini A, Kuhn M, Simonovic M, Roth A, Minguez P, Doerks T, Stark M, Muller J, Bork P, et al: The STRING database in 2011: functional interaction networks of proteins, globally integrated and scored. Nucleic Acids Res 39: D561-D568, 2011.

21. Paramasivan M, Sankaran G, Sethuraman N, Devadoss DS, Thangavelu S and Gangatharan M: Molecular modelling of urease accessory interaction proteins of Helicobacter pylori J 99 and predicting an interruption in interaction by Vigna radiata Defensins. Bioinformation 5: 410-415, 2011.

22. Feng XP, Yi H, Li MY, Li XH, Yi B, Zhang PF, Li C, Peng F, Tang CE, Li JL, et al: Identification of biomarkers for predicting nasopharyngeal carcinoma response to radiotherapy by proteomics. Cancer Res 70: 3450-3462, 2010.

23. Qu Y, Zhang H, Zhao S, Hong J and Tang C: The effect on radioresistance of manganese superoxide dismutase in nasopharyngeal carcinoma. Oncol Rep 23: 1005-1011, 2010.

24. Wei WI and Sham JS: Nasopharyngeal carcinoma. Lancet 365: 2041-2054, 2005.

25. Buettner R, Mora LB and Jove R: Activated STAT signaling in human tumors provides novel molecular targets for therapeutic intervention. Clin Cancer Res 8: 945-954, 2002.

26. Hui ZG, Tretiakova IM, Zhang ZG, Li Y, Wang XZ, Zhu JXH, Gao YH, Mai WY, Fugre K, Qian CN, Amato R, Butler B, Teh BT and Teh BS: Radiosensitization by inhibiting STAT1 in renal cell carcinoma. Int J Radiat Oncol 73: 288-295, 2009.
27. El-Hashemite N, Zhang H, Walker V, Hoffmeister KM and Kwiatkowski DJ: Perturbed IFN-gamma-Jak-signal transducers and activators of transcription signaling in tuberous sclerosis mouse models: synergistic effects of rapamycin-IFN-gamma treatment. Cancer Res 64: 3436-3443, 2004.

28 Cochet $\mathrm{O}$, Frelin C, Peyron JF and Imbert V: Constitutive activation of STAT proteins in the HDLM-2 and L540 Hodgkin lymphoma-derived cell lines supports cell survival. Cell Signal 18: 449-455, 2006.

29. Ahmed KM and Li JJ: NF-kappa B-mediated adaptive resistance to ionizing radiation. Free Radic Biol Med 44: 1-13, 2008.

30. Townsend PA, Cragg MS, Davidson SM, McCormick J, Barry S, Lawrence KM, Knight RA, Hubank M, Chen PL, Latchman DS, et al: STAT-1 facilitates the ATM activated checkpoint pathway following DNA damage. J Cell Sci 118: 1629-1639, 2005.

31. Schwarz SB, Schaffer PM, Kulka U, Ertl-Wagner B, Hell R and Schaffer M: The effect of radio-adaptive doses on HT29 and GM637 cells. Radiat Oncol 3: 12, 2008.

32. Dhandapani KM, Mahesh VB and Brann DW: Curcumin suppresses growth and chemoresistance of human glioblastoma cells via AP-1 and NFkappaB transcription factors. J Neurochem 102: 522-538, 2007.

33. Hatanpaa KJ, Burma S, Zhao D and Habib AA: Epidermal growth factor receptor in glioma: signal transduction, neuropathology, imaging, and radioresistance. Neoplasia 12: 675-684, 2007.

34. Grana TM, Sartor CI and Cox AD: Epidermal growth factor receptor autocrine signaling in RIE-1 cells transformed by the Ras oncogene enhances radiation resistance. Cancer Res 63: 7807-7814, 2003.

35. Hettinger K, Vikhanskaya F, Poh MK, Lee MK, De Belle I, Zhang JT, Reddy SA and Sabapathy K: c-Jun promotes cellular survival by suppression of PTEN. Cell Death Differ 14: 218-229, 2007.

36. Kikuchi J, Kinoshita I, Shimizu Y, Oizumi S, Nishimura M, Birrer MJ and Dosaka-Akita H: Simultaneous blockade of AP-1 and phosphatidylinositol 3-kinase pathway in non-small cell lung cancer cells. Br J Cancer 99: 2013-2019, 2008.

37. Kajanne R, Miettinen P, Tenhunen M and Leppa S: Transcription factor AP-1 promotes growth and radioresistance in prostate cancer cells. Int J Oncol 35: 1175-1182, 2009 